\title{
The Poggendorff displacement effect with only three dots
}

\author{
R. H. DAY, WENDY L. WATSON, and W. J. JOLLY \\ Monash University, Clayton, Victoria, Australia
}

\begin{abstract}
Two experiments showed that apparent displacement from exact vertical bisection, a variant of the Poggendorff effect reported by Tolansky (1964), occurs not only with three vertical lines or three dots on oblique parallels but with three vertical lines or three dots alone. The size of the effect without parallels was no different from that with parallels. The second of the two experiments, with separate groups of subjects, was a control for the possible influence of one condition on another in the first. The occurrence of the bisection form of the Poggendorff displacement effect is interpreted in terms of a perceptual compromise between exact bisection relative to the vertical and that relative to the main direction of the figure. This direction can be delineated by either parallel lines or obliquely positioned elements.
\end{abstract}

In its original form, the Poggendorff illusion is the apparent misalignment of two collinear oblique lines separated by a pair of vertical parallels which the lines intersect. The effect is evident but reduced in size when the figure is rotated so that collinear vertical lines intersect oblique parallels (Anton, 1976; Green \& Hoyle, 1964; Leibowitz \& Toffey, 1966; Weintraub \& Krantz, 1971). Tolansky (1964) demonstrated that the effect is also present when a vertical line intersecting one oblique parallel would, if extended, exactly bisect the space between two other vertical lines intersecting the other parallel. This arrangement is shown in Figure 1A. The upper vertical appears displaced to the left so that if extended downward it would not exactly bisect the space between the two lower verticals.

Recently, Day and Kasperczyk (1985) showed that this inexact-bisection form of the Poggendorff displacement effect persists when the three vertical lines are replaced by three dots coincident with the points of intersection. This arrangement is shown in Figure 1B. The upper dot is positioned so that if it were moved vertically down it would exactly bisect the space between the two lower dots. Data from two experiments indicated that the size of the inexact-bisection effect with three dots (Figure 1B) is no different from that with three lines (Figure 1A). Day and Kasperczyk then went on to show that the Poggendorff misalignment effect with two dots lying in the same vertical axis is no different from that with two lines in the same vertical axis.

Day and Kasperczyk (1985) suggested a basis for these apparent displacements, which is applicable to both apparent inexact bisection and misalignment. This explanation is couched in terms of a perceptual compromise between apparent alignment or bisection relative to the

The authors' mailing address is: Department of Psychology, Monash University, Clayton, Victoria, Australia 3168. vertical and apparent alignment or bisection relative to the direction of the parallels. This notion is clarified in Figure 2A. The upper dot is positioned so that if it were moved vertically down it would exactly bisect the space between the two lower dots. In Figure 2B, it is positioned so that if it were moved at right angles to the parallels it would exactly bisect the space between the other two. The essence of the proposed explanation is that there is a compromise in perception between exact bisection relative to the vertical and exact bisection relative to the main

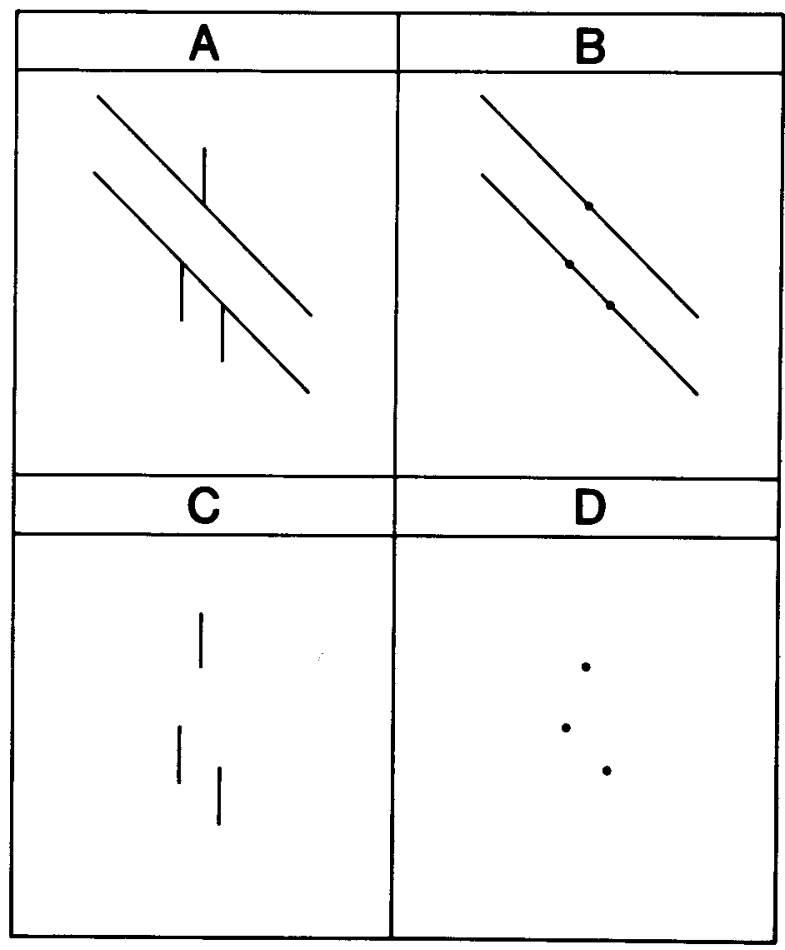

Figure 1. The four figures used in Experiment 1 and the two (A and D) used in Experiment 2. 


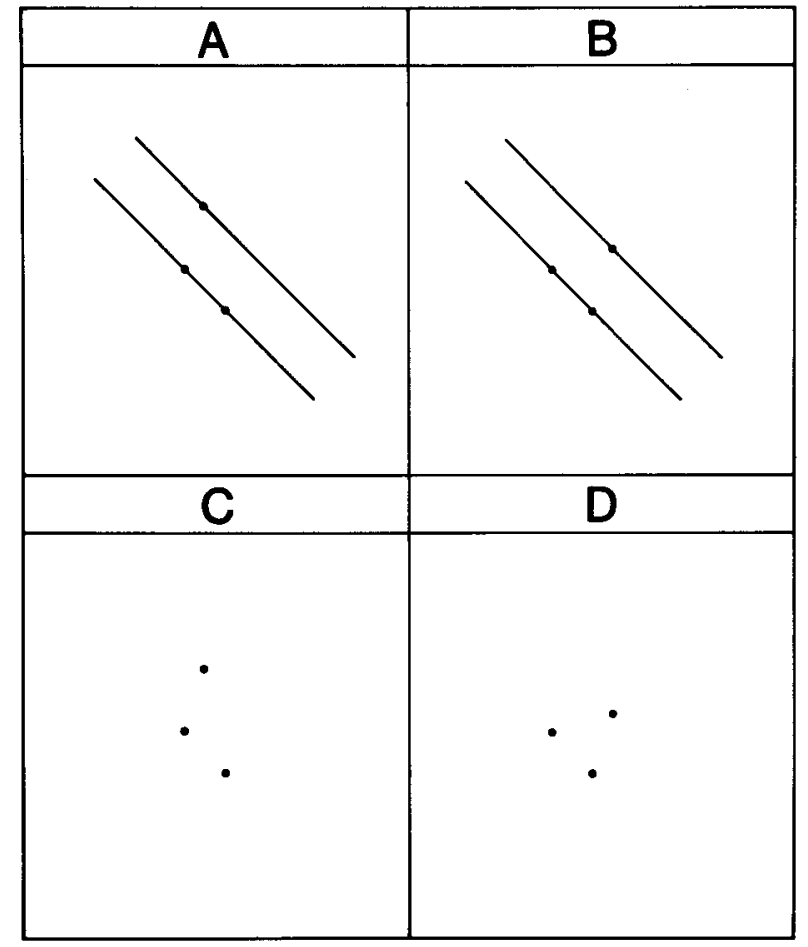

Figure 2. Exact bisection relative to the vertical and relative to the oblique direction of the figure. In $A$ and $C$, the upper dot lies on a vertical axis that exactly bisects the space between the two lower dots. In $B$ and $D$, the upper dot lies on an axis orthogonal to the direction defined by the two lower elements.

direction of the figure, in this case the axis of the parallels. The latter is conceived of as intruding upon and slightly affecting the former. Thus, when, as in Figure 2A, the upper dot is positioned on the parallel so that relative to the vertical it would exactly bisect vertically the space between the lower dots, it appears too far to the left. Consequently, to appear exactly to bisect the space vertically, it must be moved slightly along the parallel to the right, that is, in the direction of exact bisection relative to the parallels. In other words, vertical bisection in Figure 1A is compromised by bisection relative to the direction of the parallel-line figure.

If this explanation of apparent displacement is valid, it can reasonably be expected that the effect will be evident with only three vertical lines (Figure 1C) and three dots (Figure 1D). Although there are no parallels in either of these figures, an oblique direction is nevertheless clearly delineated by the lower pair of elements; a line through the midpoint of either pair would define the same oblique direction as the parallels. Casual inspection of the two figures suggests that if the upper line in Figure 1C and the upper dot in Figure 1D were, respectively, extended and moved vertically downward, neither would bisect the space between the lower pair. In each case, the upper element appears, as in Figures 1A and 1B, too far to the left for exact bisection. The two experiments described here were designed to ascertain whether these casual impressions would be sustained experimentally and, if so, the magnitude of the effect relative to that in the parallel-line figure.

The occurrence of significant apparent displacement from exact bisection in Figures 1C and 1D would not only lend support to the perceptual-compromise explanation set out above but would bear closely on two other issues concerning the Poggendorff effect. First, it could be concluded that parallels, or, indeed, any lines, are unnecessary for the occurrence of the effect. However, if the apparent displacements in Figures 1C and 1D were significant but smaller than those in Figures 1A and 1B, an additional contribution to the effect by the parallels would be indicated. Second, significant displacements with the three-line and three-dot figures would seem to rule out an explanation in terms of depth processing as proposed by Gillam $(1971,1980)$. It is highly unlikely that in either figure, and in particular in Figure 1D, the cues to depth necessary to initiate depth processing are present.

Two experiments are reported. The first, involving a within-groups design, was concerned with the occurrence and size of apparent displacements of the upper elements from exact vertical bisection in the four figures in Figure 1 . The second, involving a between-groups design, was a control for the possibility that the effect in figures with parallels (Figures 1A and 1B) transferred and influenced the effect in the figures without parallels (Figures $1 \mathrm{C}$ and $1 \mathrm{D})$.

\section{METHOD}

Since the source of subjects, apparatus, and procedure were more or less the same in these experiments as in those reported earlier (Day \& Kasperczyk, 1985), they need be described only briefly.

\section{Subjects}

The subjects were undergraduates who were paid for participation. There were 12 subjects in Experiment 1 and 20, 10 in each of two groups, in Experiment 2.

\section{Apparatus}

Stimulus figures were presented on an XY plotter (HewlettPackard 1310A) and were generated by a Z-80 microprocessor with 12-bit D/A (TM-DA100) converters. The microprocessor was programmed to present the figures in random order, randomize the direction and displacement of the starting point prior to adjustment, and record the magnitude and direction of error. A black cover with a circular aperture, $24 \mathrm{~cm}$ in diameter, was placed over the face of the display. The stimulus figures were thus presented at the center of a circular region. To reduce the brightness of the figures to a comfortable viewing level in the dimmed-out laboratory, a blue gelatin filter was placed over the display behind the aperture. Thus, the figures were bright blue in a dark field. The luminance of the figures was approximately $.6 \mathrm{~cd} / \mathrm{m}^{2}$.

The upper line or dot in the figures shown in Figure 2 could be moved in either direction along the upper parallel. Direction of movement was controlled by two directionally marked keys on a keyboard located on a table. When the keys were held down, the movement of the dot or line was smooth and continuous, and when they were tapped, it was discontinuous, moving approximately 
$.35 \mathrm{~mm}$ for each tap. After the completion of an adjustment to apparent vertical bisection, the subject pressed a third key to bring up the next figure in the series.

The viewing distance was about $114 \mathrm{~cm}$ so that $1 \mathrm{~cm}$ at the display subtended a visual angle of about $.5^{\circ}$. Head position and movement were not restrained, but subjects were instructed to keep their heads upright. This posture was monitored throughout the session.

\section{Procedure}

The subject's task was that of exact vertical bisection of the space between the elements on the lower parallel by the element on the upper parallel. The subjects were instructed to move the upper element along the parallel so that it appeared to be exactly halfway between the two lower ones with respect to the vertical, that is, so that if it were to be moved down vertically it would exactly bisect the space between the two lower elements. Adjustments were made by either holding down the appropriate key to produce continuous movement along the parallel or by pressing it to produce discrete $(.35-\mathrm{mm})$ steps. Either mode of adjustment was permitted at any stage.

A trial consisted of movement of the upper element from a point of obvious inexact bisection to one of apparently exact bisection. The starting position was from the left or right of the physically correct position. There were six trials for each figure, two practice trials and four experimental trials. The starting points for the practice and experimental trials were differently randomized for each subject. The measure was always the horizontal displacement, in millimeters, of the upper line or dot from exact vertical bisection of the space between the two lower lines or dots. Displacements in the direction to compensate for the illusion were treated as positive and those in the opposite direction as negative. Only the four experimental trials following the two practice trials were measured, and the mean of these was the score for the subject.

The session began with instructions during which the stimulus figures and methods of adjustment by keypressing were demonstrated. There were no time limits to making adjustments, and the subjects proceeded at their own pace, bringing up a new figure after an adjustment had been completed. The subjects were told, during the instruction period, how many adjustments they would be required to make.

\section{EXPERIMENT 1}

As pointed out above, a line or dot on an oblique parallel appears to be displaced from its position of exact vertical bisection of the space between a pair of lines or dots on another parallel (Figures 1A and 1B). The purpose of the first experiment was to find out whether the same effect occurs without parallels, with the oblique direction delineated only by the oblique arrangement of the pair of elements, and, if so, whether the size of the effect without parallels is different from that with parallels.

\section{Stimulus Figures}

The four stimulus figures are shown in Figure 1. The lines were $.8 \mathrm{~mm}$ thick, and the dots were $1 \mathrm{~mm}$ in diameter. The $45^{\circ}$ parallels were $80 \mathrm{~mm}$ long and $15 \mathrm{~mm}$ apart. The vertical lines in Figures $1 \mathrm{~A}$ and $1 \mathrm{C}$ were $15 \mathrm{~mm}$ long. The pairs of lines or dots on the lower parallels were $15 \mathrm{~mm}$ apart along the parallels. The arrangements of the lines and dots without parallels (Figures 1C and 1D) were identical to those with parallels (Figures $1 \mathrm{~A}$ and $1 \mathrm{~B}$ ).

\section{Results and Discussion}

The mean displacements, in millimeters, from positions of exact vertical bisection, each derived from 12 individual mean scores based on four adjustments, are shown
Table 1

Mean Displacements (in Millimeters) from Exact Vertical Bisection Together with Standard Deviations for the Stimulus Figures (Figure 1) in Experiments 1 and 2

\begin{tabular}{lrcrr}
\hline & \multicolumn{4}{c}{ Figures } \\
\cline { 2 - 5 } & $\mathrm{A}$ & $\mathrm{B}$ & $\mathrm{C}$ & $\mathrm{D}$ \\
\hline & \multicolumn{4}{c}{ Experiment 1} \\
Mean & 1.27 & 1.58 & 1.04 & 1.42 \\
SD & .79 & 1.31 & .62 & 1.35 \\
& & Experiment 2 & & \\
Mean & 1.17 & - & - & .88 \\
SD & .86 & - & - & .64 \\
\hline
\end{tabular}

together with their standard deviations in Table 1. It can be noted that the four mean displacements were of about the same order of magnitude, ranging from $1.04 \mathrm{~mm}$ for the vertical lines without parallels to $1.58 \mathrm{~mm}$ for the dots on the parallels. It can also be noted that of the 12 individual means, 1 was negative for Figure 1A, 2 were for Figure 1B, none were for Figure 1C, and 2 were for Figure 1D. A two-way ANOVA, in which the factors were parallel lines (present or absent) and type of element (vertical lines or dots), showed that neither factor was significant [parallels, $F(1,11)=1.92, p>.10$; element, $F(1,11)=2.60, p>.10]$. The interaction between the two factors fell far short of achieving significance $(F<1.0)$. Four separate $t$ tests were carried out in order to ascertain whether the means for each of the four figures were significantly different from zero. All four proved to be so [Figure 1A, $t(11)=5.58, p<.0003$; Figure 1B, $t(11)=4.17, p<.002$; Figure $1 \mathrm{C}, t(11)=$ $5.81, p<.0002$; Figure $1 \mathrm{D}, t(11)=3.65, p<.004$ ]

In summary, although the four mean displacements from exact bisection for the figures shown in Figure 1 were themselves significant, they were not significantly different from each other. Neither the presence of the parallels nor the type of element affected the size of the displacements.

These results are quite clear in showing that the effect does occur with both three vertical lines and with three dots, and that with both types of element the effect is not different in size from that with parallels. One impediment to accepting these conclusions unreservedly is the possibility that, with all subjects serving under all conditions, displacement with parallels influenced displacement without parallels. This possibility was the subject of the second experiment.

\section{EXPERIMENT 2}

As in the earlier series of experiments, it was conceivable that the inexact bisection effect in one condition could have influenced that in another. Specifically, it was possible that the effect in the figures with parallels could have exercised an influence in those without parallels. Although no interfigure influence was found in the earlier series, it was, nevertheless, thought prudent to investigate the possibility again. In the second experiment, therefore, one group of 10 subjects was assigned to Figure 1A, the origi- 
nal Tolansky figure, and another group of 10 subjects was assigned to Figure 1D, the three-dot figure. If there were no interfigure influences, the effect with both figures could be expected to be significantly different from zero but not significantly different from each other as in Experiment 1.

The two stimulus figures are shown in Figures 1A and 1D. They were the same as those included in Experiment 1 .

\section{Results and Discussion}

The mean displacements from exact bisection are shown with their standard deviations in Table 1 . It can be noted that the mean for Figure 1A is about the same size as that in Experiment 1, whereas that for Figure 1D is smaller. Of the 12 individual means for Figure $1 \mathrm{~A}$, all were positive; of those for Figure 1D, 10 were positive and 2 negative. Separate $t$ tests indicated that both means were significantly different from zero [Figure $1 \mathrm{~A}, t(11)=4.32$, $p<.002$; Figure 1D, $t(11)=4.31, p<.002$ ]. A third $t$ test was carried out to ascertain whether the difference between the two means was significant. It proved not to be $[t(11)=.788, p=.45]$.

The smaller mean for Figure 1D notwithstanding, the outcomes of the second experiment are in accord with those of the first. With separate groups of subjects, apparent displacement with three dots without parallels is significant and as great as that in the original Tolansky (1964) figure, consisting of three lines intersecting oblique parallels. As in the earlier series, it can be concluded with confidence that, for a within-groups experiment, apparent displacement in one figure does not influence that in another.

\section{GENERAL DISCUSSION}

It is to be emphasized that the Tolansky (1964) inexactbisection effect in Figure 1A is essentially the same as the Poggendorff misalignment effect. Apparent displacement of lines intersecting a pair of parallels is manifested in inexact bisection rather than in apparent misalignment. It is the task that is different. The earlier experiments showed that both these forms of apparent displacement occur with dots on oblique parallels. Furthermore, the size of the displacement with dots on the parallels was as great as that with vertical lines intersecting them. This result serves straightaway to rule out numerous theories based on changes in the apparent orientation of lines, apparent size of angles formed by lines and parallels, and apparent distance between parallels due to Müller-Lyer-type effects. The result also rules out explanations based on blurring of apexes and seriously impugns those couched in terms of depth processing initiated by cues to depth that are implicit in the figure.

The occurrence of apparent displacements in figures consisting of only three vertical lines (Figure 1C) or three dots (Figure 1D) with the same magnitude as in figures consisting of lines intersecting parallels (Figure 1A) and dots on parallels (Figure 1B) strengthens these earlier conclusions. In particular, the outcomes of Experiments 1 and 2 call seriously into question depth processing as the basis of apparent displacement. Implicit cues for depth that lead to depth processing cannot reasonably be attributed to a figure consisting simply of three vertical lines or three dots.

At the same time, the data from these experiments are in accord with the explanation of apparent displacement adumbrated above, as will be apparent from Figures $2 \mathrm{C}$ and 2D. In Figure 2C, the upper dot is located on the axis of exact vertical bisection of the space between the lower pair. In Figure 2D, it is located on the axis of bisection orthogonal to the oblique direction of the lower pair. If apparent vertical bisection represents a perceptual compromise between the vertical axis and the axis orthogonal to that of the figure, that is, the oblique direction of the figure, it would be expected that the upper dot would have to be moved to the right. That is, in fact, what occurs.

In summary, the occurrence of apparent displacement of the same size as that in a parallel-line figure in one consisting of only three vertical lines or three dots confirms the inadequacy of numerous earlier explanations of the Poggendorff effect. The explanation proposed earlier (Day \& Kasperczyk, 1985) in terms of a compromise between bisection (or alignment) relative to one direction and that relative to the direction defined by the figure can account for both the earlier results and those reported here. It now remains to examine the adequacy of this explanation in relation to the now considerable body of data on apparent displacement in other figures and to test predictions from it. In particular, it is of interest to establish whether apparent bisection relative to the direction defined by the figure is a compromise with bisection relative to the vertical when the latter is clearly delineated.

\section{REFERENCES}

AN'TON, B. S. (1976). Poggendorff illusion as a function of orientation of transversal and parallel lines. Perceptual \& Motor Skills, 43, 83-90.

DAY, R. H., \& KASPERCZYK, R. T. (1985). Apparent displacement of lines and dots in a parallel-line figure: A clue to the basis of the Poggendorff effect. Perception \& Psychophysics, 38, 74-80.

Gillam, B. (1971). A depth processing theory of the Poggendorff illusion. Perception \& Psychophysics, 10, 211-216.

Gillam, B. (1980). Visual illusions. Scientific American, 242, 102-112.

GreEN, R. T., \& Hoyle, E. M. (1964). The influence of spatial orientation on the Poggendorff illusion. Acta Psychologica, 22, 348-366.

Leibowitz, H., \& TofFEY, S. (1966). The effect of rotation and tilt on the magnitude of the Poggendorff illusion. Vision Research, 6, 101-103.

Tolansky, S. (1964). Optical illusions. London: Pergamon.

Weintraub, D. J., \& Krantz, D. H. (1971). The Poggendorff illusion: Amputations, rotations, and other perturbations. Perception \& Psychophysics, 10, 257-264.

(Manuscript received August 5, 1985; revision accepted for publication February 26, 1986.) 